16

\title{
Выделение полос поглощения мономеров и агрегатов в спектре слоя цианинового красителя и определение ориентации молекул
}

\author{
(C) Е.Н. Калитеевская, В.П. Крутякова, Т.К. Разумова, А.А. Старовойтов \\ Университет ИТМО, \\ 197101 Санкт-Петербург, Россия \\ ฯ e-mail: anton.starovoytov@gmail.com
}

Поступила в редакцию 05.02.2018 г.

\begin{abstract}
При исследовании молекулярных слоев или органических тонких пленок, возникает задача выделения полос отдельных мономеров или агрегатов из спектра поглощения многокомпонентной структуры. Помимо концентрации компонентов, на оптические свойства таких образцов влияет наклон молекул относительно поверхности подложки; молекулярная ориентация может быть определена из спектров дихроичного отношения при наклонном зондировании образцов. В работе на примере слоев трикарбоцианинового красителя проведено сравнение двух независимых методов выделения спектров компонентов из спектра поглощения слоя: посредством сравнения спектров слоев разной толщины и на основе спектров дихроичного отношения. Изучено воздействие лазерного излучения на образцы, которое приводит к изменению дихроичного отношения. Проведен расчет фотоиндуцированных изменений углов наклона молекул, который позволил уточнить диапазон возможных значений этих углов.
\end{abstract}

DOI: $10.21883 /$ OS.2018.09.46560.31-18

\section{Введение}

В настоящее время органические молекулы являются важным структурным элементом в различных оптоэлектрических устройствах, таких как светоизлучающие диоды [1-4], фотовольтаические ячейки [5-7] и полевые транзисторы $[8,9]$. В частности, большое внимание уделяется изучению оптической нелинейности органических молекул, потому что высокий нелинейный отклик, характерный для таких молекул, является основополагающим для реализации оптических переключателей, которые будут использованы в высокоскоростных оптических сетях нового поколения. Для большинства этих применений цианиновые молекулы являются перспективными благодаря своим оптическим характеристикам, таким как настраиваемая длина волны поглощения и сверхбыстрый отклик [10-12]. Однако для реализации реальных устройств до сих пор остается проблема методики создания тонкопленочных образцов, которые имеют необходимые оптические свойства. Это связано с тем, что при высаживании на поверхность молекулы формируют различные изомеры и агрегаты, что сказывается, например, на процессах переноса энергии.

В работах $[13,14]$ нами было предложено и обсуждалось разделение на отдельные компоненты спектров поглощения молекулярных слоев цианиновых красителей на диэлектрических подложках. Были измерены спектры поглощения слоев для большого числа молекул различного строения, нанесенных методом spin-coating на стеклянные подложки из этанольных растворов разной концентрации. Методика нанесения слоев подробно описана в [15]. Спектры поглощения слоев симметричных цианинов заметно уширены по сравнению со спектрами этих же молекул в растворе, так как состоят из нескольких компонентов с отличающимися спектрами поглощения. Полное число различных типов мономерных компонентов в слоях разной толщины зависит от длины цепи сопряжения молекулы (от числа углеродных связей в цепи), наличия стерических затруднений и от толщины слоя [14]. Было показано, что в спектрах многослойных образцов на длинноволновом и коротковолновом краях достаточно однозначно выделяются спектры молекулярных ассоциатов $-J$-агрегатов и димеров $[13,14]$. В работах $[13,14,16]$ нами были приведены доказательства стереоизомерной природы мономерных компонентов в многослойных образцах. Для большинства исследованных симметричных цианиновых молекул полоса длинноволнового компонента слоя близка по положению к полосе поглощения этих молекул в этанольных растворах, где присутствует только один стереоизомер (all-trans-конфигурация в стерически незатрудненных молекулах) [17].

Выделение спектров компонентов слоев проводилось на основании исследования концентрационных зависимостей экспериментальных спектров поглощения в гомологических рядах цианиновых красителей. Спектр каждого компонента аппроксимировался суммой гауссовых контуров, описывающих электронные переходы из основного состояния $S_{0}$ в разные колебательные подуровни возбужденного состояния $S_{1}$. Параметры гауссовых контуров мономеров определялись из спектров моно- и субмонослоев. Сравнение суммарного спектра поглощения мономеров с экспериментальным спектром поглощения молекулярного слоя позволяло выделить спектры поглощения ассоциированных форм [14].

В работах $[18,19]$ показано, что дипольные моменты переходов с поглощением как мономеров, так и ассоциатов в слоях цианиновых красителей наклонены 


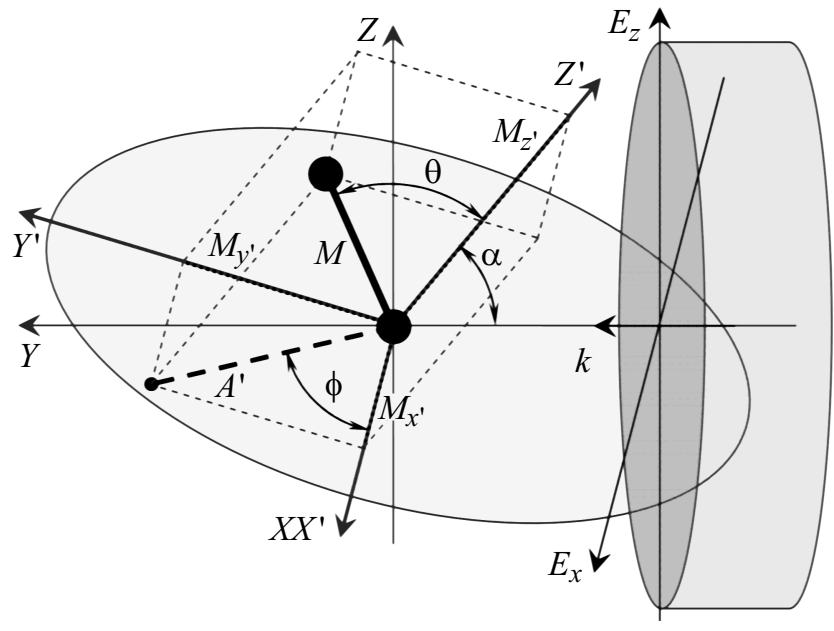

Рис. 1. Схема зондирования образцов для определения ориентации молекулы по спектрам дихроичного отношения.

по отношению к вертикали к плоскости подложки на отличающиеся углы. Эти углы меняются при облучении слоев импульсным лазерным излучением в пределах спектра поглощения слоя - увеличиваются у дипольных моментов переходов мономеров и $J$-агрегатов и уменьшаются у димеров. Оценки углов наклона $\theta$, где $\theta-$ угол между перпендикуляром к плоскости подложки и вектором дипольного момента перехода с поглощением для молекулярных компонентов слоя, делались по результатам исследования спектров дихроичного отношения слоев $\left(R=D_{z} / D_{x}\right)$, где $D_{z}$ и $D_{x}$ - плотности поглощения слоя при наклонном (угол $\alpha$ на рис. 1) зондировании линейно поляризованным излучением при двух ортогональных ориентациях вектора Е. Отметим, что в молекулах мономеров и $J$-агрегатов направление дипольного момента перехода с поглощением совпадает с цепью молекулы и тем самым отражает расположение самой молекулы на подложке. Исключение составляют димеры, у которых дипольный момент перехода может быть представлен векторной суммой переходов в составляющих его мономерах [20-22].

На рис. 1 представлена схема спектральных измерений дихроичного отношения. Система координат $X Y Z$ привязана к осям спектрального прибора. Линейно поляризованное зондирующее излучение распространяется вдоль оси $Y$, электрический вектор $\mathbf{E}$ излучения совпадает либо с вертикальной осью $Z$ (в дальнейшем - вертикальная поляризация), либо с горизонтальной осью $X$ (горизонтальная поляризация). $X^{\prime} Y^{\prime} Z^{\prime}-$ система координат слоя. Оси $X^{\prime}$ и $Y^{\prime}$ лежат в плоскости, параллельной плоскости подложки слоя, ось $Z^{\prime}$ перпендикулярна к поверхности подложки. Оси $X^{\prime}$ и $X$ совпадают. Изменение угла падения зондирующего луча $\alpha$ производится поворотом слоя вокруг оси $X^{\prime}$. Ориентация дипольного момента оптического перехода $S_{0} \rightarrow S_{1}$ мономерного компонента (угол $\theta$ относительно оси $Z^{\prime}$ ) совпадает с ориентацией цепи сопряжения молекулы М. Проек- ции вектора дипольного момента на плоскость $X^{\prime} Y^{\prime}$ (т.е. $M_{i}^{\prime}$ ) имеют круговую симметрию распределения вокруг оси $Z^{\prime}$.

Для линейно поляризованного излучения вероятность оптического перехода с поглощением пропорциональна квадрату проекции вектора $\mathbf{E}$ излучения на вектор дипольного момента перехода М. Для взаимного расположения векторов М и $\mathbf{E}$ на рис. 1 оптические плотности для двух ориентаций вектора $\mathbf{E}$ при угле падения $\alpha$ могут быть записаны следующим образом:

$$
\begin{gathered}
D_{x}(\lambda, \varphi, \alpha)=\varsigma \sin ^{2} \theta \cos ^{2} \varphi, \\
D_{z}(\lambda, \varphi, \alpha)=\varsigma\left(\cos ^{2} \theta \sin ^{2} \alpha+\cos ^{2} \alpha \sin ^{2} \theta \sin ^{2} \varphi\right),
\end{gathered}
$$

где коэффициент $\varsigma$ отражает сечение перехода с поглощением на определенной длине волны, а также поверхностную концентрацию молекул. Предполагая узкое распределение углов пространственной ориентации $\theta$ всех молекул рассмотренного компонента, и в соответствии с круговой симметрией ориентации молекул относительно нормали к поверхности подложки зависимости оптической плотности поглощения слоя $D$ от углов $\theta$ и $\alpha$ для ориентаций $\mathbf{E}$ параллельно $Z$ и $X$ равны:

$$
\begin{aligned}
D x \sim \int_{0}^{2 \pi} D_{x} & (\lambda, \varphi, \alpha) d \varphi \sim \int_{0}^{2 \pi}\left[\varsigma \sin ^{2} \theta \cos ^{2} \varphi\right] d \varphi=\varsigma \pi \sin ^{2} \theta \\
D z & \sim \int_{0}^{2 \pi} D_{z}(\lambda, \varphi, \alpha) d \varphi \sim \int_{0}^{2 \pi}\left[\varsigma \left(\cos ^{2} \theta \sin ^{2} \alpha\right.\right. \\
& \left.\left.+\cos ^{2} \alpha \sin ^{2} \theta \sin ^{2} \varphi\right)\right] d \varphi \\
& =\varsigma \pi\left(\cos ^{2} \alpha \sin ^{2} \theta+2 \cos ^{2} \theta \sin ^{2} \alpha\right) \\
& =\varsigma \pi\left[\cos ^{2} \alpha+\cos ^{2} \theta\left(2-3 \cos ^{2} \alpha\right)\right] .
\end{aligned}
$$

Таким образом, дихроичное отношение позволяет определить угол наклона молекул однокомпонентного слоя:

$$
R=D z / D x=\frac{\cos ^{2} \alpha+\cos ^{2} \theta\left(2-3 \cos ^{2} \alpha\right)}{\sin ^{2} \theta} .
$$

Величина $R$ для однокомпонентного слоя не зависит от длины волны в пределах исследуемой полосы поглощения компонента, т. е. значения $\theta$ можно определять по отношению $D_{z}$ и $D_{x}$ для любой $\lambda$ в пределах полосы поглощения компонента. Отсюда следует, что неизменность величины $R$ в пределах всей полосы поглощения слоя свидетельствует о наличии в слое компонентов с близкими углами ориентации, в то время как спектральный ход $R$ говорит о присутствии компонентов с различными углами ориентации.

Для слоя, содержащего молекулярные компоненты только одного типа, угол наклона определяется следующим отношением:

$$
\cos ^{2} \theta=\frac{R-\cos ^{2} \alpha}{R+2-3 \cos ^{2} \alpha} .
$$


Отметим, что при $\alpha=0$ значение $R=1$ и величина $\cos ^{2} \theta$ оказывается не определенной $0 / 0$. Таким образом, определение угла ориентации из этого выражения при нормальном падении невозможно. В нашей работе определение $\theta$ осуществляется при четырех углах падения зондирующего излучения $\alpha=30^{\circ}, 45^{\circ}, 52.5^{\circ}, 60^{\circ}$, затем результаты расчета усредняются по углам $\alpha$.

Дихроичное отношение для n-компонентного слоя:

$$
R=\frac{\sum_{n} \frac{D_{n}}{\sin ^{2} \theta_{n}}\left(\cos ^{2} \alpha+\cos ^{2} \theta_{n}\left[2-3 \cos ^{2} \alpha\right]\right)}{\sum_{n} D_{n}}
$$

где $D_{n}-$ плотности поглощения отдельных $n$-компонентов слоя при $\alpha=0$.

В работах $[18,19]$ углы $\theta_{n}$ определялись в отдельных точках спектра. Для оценки величин $\theta_{n}$ для разных компонентов слоя авторами использовались следующие методики.

1. Определялся угол $\theta_{n}$ в той области спектра, где отсутствует перекрытие полос поглощения выделенных компонентов (обычно это самая длинноволновая область спектра поглощения слоя) по формуле (4). Затем вычисленные значения $\theta_{n}$ подставлялись в формулу (5) для вычисления угла наклона $\theta_{i}$ других (обычно более коротковолновых) компонентов. Такие вычисления проводились для длины волны $\lambda$ в области перекрытия спектров двух компонентов. При этом учитывались соотношения оптических плотностей $D_{n}$ компонентов на $\lambda$.

2. В нормированном спектре поглощения слоя выделялись отстоящие друг от друга точки спектра на определенной длине волны, значительно различающиеся значениями $D_{n}$ компонентов. Для каждого спектрального диапазона определялись углы $\theta_{n}$ из системы $n$ уравнений (5) для $n$ перекрывающихся компонентов слоя.

Обе эти методики оценки углов $\theta_{n}$ в области перекрытий спектров поглощения компонентов предполагают включение в уравнения соотношения их оптических плотностей. При этом вопрос о правильности разделения спектров слоев на спектры отдельных молекулярных компонентов возникает достаточно часто. Этот вопрос особенно существен в случае многослойных образцов, где присутствует несколько молекулярных форм, поскольку возможен некоторый произвол в определении спектральных параметров этих форм. Как видно из вышесказанного, между соотношениями плотностей поглощения компонентов в каждой точке их перекрывающихся спектров и углами их наклона существует зависимость, определяемая формулой (1). При этом углы наклона должны оставаться неизменными во всей области поглощения каждого компонента ${ }^{1}$. $\mathrm{B}$ настоящей работе предпринята попытка рассчитать отдельные участки спектров поглощения компонентов

\footnotetext{
${ }^{1}$ Скорее всего внутри каждой молекулярной формы слоя существует некоторое распределение в значениях этих углов, и поляризационные спектры отражают их среднее значение.
}

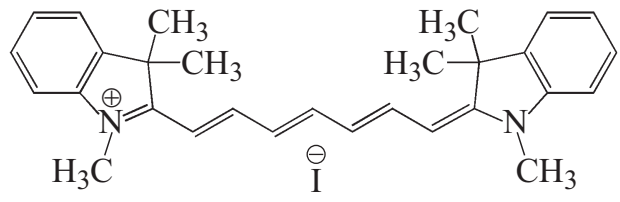

Рис. 2. Структура молекулы НІТСІ.

слоя исследуемой молекулы, исходя из независимых экспериментальных результатов: измеренного спектра поглощения молекулы при нормальном падении зондирующего излучения на подложку и спектра дихроичного отношения $R_{i}$, полученного в пределах всего спектра поглощения слоя с шагом 10 nm при наклонном положении подложки по отношению к зондирующему излучению $\left(\alpha=60^{\circ}\right)$. При этом варьировались углы $\theta$ и оценивались пределы соответствующих возможных ошибок в разделении спектров компонентов.

\section{Образцы исследования}

В качестве молекулы для исследования был выбран $1,3,3,1^{\prime} 3^{\prime} 3^{\prime}$-гексаметилиндотрикарбоцианин йодид (НITCI), структура которого приведена на рис. 2. Два образца толстых слоев ( 40 условных монослоев) на стеклянных подложках были получены по методике spin-coating [15]. В спектрах поглощения образцов толстых слоев было выделено одинаковое количество молекулярных компонентов. Образец 1 облучался моноимпульсами рубинового лазера длительностью $12 \mathrm{~ns}$ со средней плотностью энергии $17 \mathrm{~mJ} / \mathrm{cm}^{2}$, образец 2 - со средней плотностью энергии $50 \mathrm{~mJ} / \mathrm{cm}^{2}$. Во втором случае суммарная плотность энергии облучения была существенно больше, чем в первом, и соответственно изменения спектров дихроичного отношения были гораздо ярче выражены.

Нормированные спектры поглощения необлученного образца 1 и облученного образца 2 , а также спектры дихроичного отношения $\left(R_{60}, R_{52.5}, R_{45}, R_{30}\right)$ при углах наклона образца $\alpha=60^{\circ}, 52.5^{\circ}, 45^{\circ}, 30^{\circ}$ представлены на рис. $3, a$ и $3, b$ соответственно. На рисунках обозначены полосы поглощения молекулярных компонентов: $F_{1}$ - all-trans-изомер, $F_{2}$ и $F_{3}-c i s$-изомеры разного строения, $F_{j}-J$-агрегат, $F_{d}-$ димер.

\section{Выделение полос компонентов и определение углов ориентации}

Для расчета спектров форм были выбраны спектральные участки, где величины $R_{i}$ меняются достаточно сильно, что свидетельствует о перекрытии спектров поглощения компонентов слоя и значительном различии в ориентациях поглощающих компонентов относительно подложки. Такие резкие изменения спектра дихроичного отношения наблюдаются на длинноволновом 
$(\sim 800-890 \mathrm{~nm})$ и коротковолновом $(\sim 550-620 \mathrm{~nm})$ участках спектра и характерны для всех толстых слоев исследованных нами цианиновых красителей. Причина столь резких изменений спектра дихроичного отношения в многослойных образцах - возникновение ассоциированных молекулярных форм с углами ориентации дипольных моментов перехода с поглощением, сильно отличающимися от соответствующих углов мономеров. Следует отметить, что в тонких моно- и субмономолекулярных слоях, где отсутствуют ассоциированные молекулярные формы, спектральные зависимости дихроичного отношения при разных углах наклона подложки практически параллельны оси длин волн [23], и величины $R$ мало меняются на протяжении всей области поглощения слоя, что свидетельствует о близости углов ориентации дипольных моментов перехода мономерных форм.

Как следует из формулы (4), в точках на спектре поглощения слоя, где присутствует полоса только одного компонента, угол $\theta$ ориентации дипольного момента перехода с поглощением для этого компонента может быть непосредственно определен по значениям дихроичного отношения $R$ и углов наклона образца $\alpha$, и точность этой оценки не зависит от разделения спектра на спектры компонентов.

По условиям выделения спектров отдельных форм в многокомпонентном слое сумма спектров компонентов в области их перекрытия должна совпадать с экспериментально измеренным спектром молекулярного слоя $D$. Это дает возможность достаточно надежно оценить углы ориентации в отдельных точках, где вклад спектров других компонентов по крайней мере мал, в частности, на длинноволновом краю молекулярного спектра (область поглощения $J$-агрегатов). В области перекрытия спектров двух компонентов знание соотношений их оптических плотностей при нормальном падении и угла ориентации одного из них дает возможность оценить угол ориентации второго. Результат расчета углов ориентации компонентов по дихроичному отношению в отдельных точках спектра представлен в табл. 1.

Максимальная средняя квадратичная погрешность этих измерений при трех значениях углов ориентаций слоя не превышает $1^{\circ}$. Однако при измерениях углов $\theta$ в отдельных точках на малом участке спектра в оценке может присутствовать неизвестная систематическая ошибка - в частности, при измерении спектров величин $D_{z}$ и $D_{x}$, а также при определении дихроичного отношения $R=D_{z} / D_{x}$ (вследствие возможных неравномерностей толщины образца в пределах всей его площади и изменение зондируемой площади образца при изменении углов $\alpha$ ). Оценка углов по описанным выше методикам для многочисленных облученных и необлученных молекулярных слоев дает основания полагать, что в данном случае эта ошибка не превышает $2^{\circ}-3^{\circ}$. В частности, на малую суммарную погрешность данных оценок указывает пересечение в одной точке спектров дихроичного отношения на рис. 3, измеренных при разных значениях угла $\alpha$. Такой результат следует из формулы (4) при $R=1$.

На рис. 3 представлены как результаты разделения спектров поглощения необлученного образца 1 и облученного образца 2 на спектры отдельных компонентов по описанным выше методикам, так и результаты расчета их отдельных участков по формуле (5) на основе измерений спектра дихроичного отношения. В табл. 1 приведены допустимые интервалы углов ориентации компонентов и соответствующие оптимизированные значения, при которых наблюдалось максимальное совпадение расчетных участков спектров с проведенным выделением спектров компонентов. Участки спектров $F_{1}$ или $F_{3}$ в области их пересечений со спектрами $F_{j}$ или $F_{d}$ соответственно (рис. 3) определялись из уравнения (6) как разность между спектром поглощении слоя и спектрами $F_{j}$ или $F_{d}$ :

$$
\begin{aligned}
& R_{60}=\frac{D-D_{n}}{\sin ^{2} \theta_{n}}\left(\sin ^{2} \alpha\left[1-\sin ^{2} \theta_{n}\right]+0.5 \cos ^{2} \alpha \sin ^{2} \theta_{n}\right) \\
& +\frac{D_{n}}{\sin ^{2} \theta_{m}}\left(\sin ^{2} \alpha\left[1-\sin ^{2} \theta_{m}\right]+0.5 \cos ^{2} \alpha \sin ^{2} \theta_{m}\right),
\end{aligned}
$$

где $R_{60}$ - дихроичное отношение при угле наклона $\alpha=60^{\circ}, D-$ спектр поглощения слоя, $D_{n}-$ спектр отдельного компонента $\left(F_{j}\right.$ или $\left.F_{d}\right), \theta_{n}-$ угол наклона дипольного момента перехода формы $F_{j}$ или $F_{d}, \theta_{m}-$ угол наклона дипольного момента перехода формы $F_{1}$ или $F_{3}$.

Еще раз подчеркнем, что исходные оценки углов $\theta$ на тех участках спектра, где присутствует только одна форма, а вклад других форм в поглощение слоя заведомо отсутствует или крайне мал, зависят только от дихроичного отношения $R$. В данном случае у обоих исследованных слоев такой участок существует на самом длинноволновом конце спектра в области поглощения $J$ агрегатов, где изменения характера спектра дихрочного отношения ярко выражены. В связи с этим оценка $\theta_{j}$ представляется нам достаточно надежной во всей области поглощения $J$-агрегатов. В то же время в коротковолновой области спектра слоя, где также наблюдается резкое изменение спектральной зависимости $R$, оценка $\theta_{d}$ только по спектру дихроичного отношения оказывается некорректной в силу полного перекрытия спектров $F_{d}$ и $F_{3}$. Вопрос о характере спектров и возможной вариации углов в этой области обсуждается ниже.

Предварительные оценки $\theta_{1}$ и $\theta_{3}$ в областях поглощения all-trans-изомера $F_{1}$ и мономерной формы $F_{3}$, приведенные в табл. 1, выполнены для отдельных точек спектров этих форм по описанным выше методикам. В то же время очевидно, что эти величины должны оставаться неизменными в пределах спектра поглощения каждой компоненты. В предположении правильности оценок $\theta_{j}$ характер рассчитанного длинноволнового участка спектра сильно зависит от значений угла $\theta_{1}$. 
Таблица 1. Углы ориентации компонентов молекулярных слоев исследованных образцов

\begin{tabular}{c|c|c|c|c|c|c|c|c}
\hline \multicolumn{7}{c|}{ Необлученный образец 1} & \multicolumn{5}{c}{ Облученный образец 2} \\
\hline$\theta_{j}, \mathrm{deg}$ & $\theta_{1}, \mathrm{deg}$ & $\theta_{3}, \mathrm{deg}$ & $\theta_{d}, \mathrm{deg}$ & $\theta_{j}, \mathrm{deg}$ & $\theta_{1}, \mathrm{deg}$ & $\theta_{3}, \mathrm{deg}$ & $\theta_{d}, \mathrm{deg}$ \\
\hline \multicolumn{7}{c}{ Расчет углов ориентации компонентов по дихроичному отношению } \\
\hline 61 & 53.5 & 49 & 71 & 67 & - & $\leq$ \\
\hline 67 & $61-63$ & $54-58$ & $45-49$ & $73-75$ & $65-67$ & $56-59$ & $\leq 30$ \\
\hline 67 & 61 & 54 & 47 & 73 & 67 & 55 & $\leq 30$ \\
\hline
\end{tabular}

Примечание. Прочерк означает, что угол ориентации определить нельзя.

В табл. 1 представлены оптимизированные значения углов, при которых рассчитанные спектры компонентов наилучшим образом совпадают со спектрами, построенными на основании концентрационных зависимостей. Очевидно, что эти значения близки, так как при расчетах углов наклона компонентов в отдельных точках спектра обычно требовалось учитывать соотношения величин $D_{n}$ компонентов в данной точке. В связи с этим в проведенном нами расчете угла $\theta_{1}$, значения углов $\theta_{3}$ и $\theta_{d}$ варьировались в широких пределах. При этом оценивались отклонения спектров поглощения компонентов от спектров, построенных на основании концентрационных зависимостей. Сразу отметим, что для необлученного слоя (рис. 3,a) при взаимных вариациях $\theta_{j}$ и $\theta_{1}$ в пределах ошибок измерений обычно можно добиться относительно хорошего совпадения расчетного спектра с результатом независимого разделения спектра слоя на спектры отдельных форм. Однако эти вариации не превышают $2^{\circ}$. Также отметим, что уменьшение или увеличение $\theta_{j}$ больше, чем на $2^{\circ}$, приводит соответственно или к выходу спектра $F_{j}$ за пределы спектра слоя, или сильному искажению его формы и сдвигу максимума поглощения в коротковолновую область, что не соответствует характеру спектра дихроичного отношения. Этот результат подтверждает надежность измерений $\theta_{j}$ для всей области поглощения формы $F_{j}$. На рис. 3, $a$ показано максимально возможное отклонение рассчитанных участков спектра компонентов образца 1 от исходного разделения на спектры форм при неизменном значении $\theta_{j}$ и вариации угла $\theta_{1}$ в диапазоне $2^{\circ}$ (табл. 2 ). Дальнейшее увеличение $\theta_{1}$ больше, чем на $1^{\circ}$ приводит к отрицательным значениям интенсивности поглощения $F_{j}$ и к выходу спектра $F_{1}$ за пределы спектра слоя, а уменьшение $\theta_{1}$ больше, чем на $2^{\circ}-$ к маловероятному сдвигу максимума поглощения $F_{j}$ в коротковолновую область. На рис. 3, $b$ показаны возможные варианты расчетных участков спектров $F_{j}$ и $F_{1}$ облученного образца 2 при разных $\theta_{j}$ и $\theta_{1}$ (табл. 2).

Аналогичные расчеты, проведенные для коротковолновой области поглощения слоя $(550-600 \mathrm{~nm}$, рис. $3, a)$
Таблица 2. Значения углов ориентации, при которых производились расчеты спектров поглощения компонентов

\begin{tabular}{c|c|c|c|c|c|c}
\hline № & $\theta_{1}, \operatorname{deg}$ & $\theta_{j}, \operatorname{deg}$ & $\theta_{3}, \operatorname{deg}$ & $\theta_{d}, \operatorname{deg}$ & Образец & Рисунок \\
\hline 1 & 61 & 67 & - & - & 1 & $3, a$ \\
2 & 63 & 67 & - & - & 1 & $3, a$ \\
3 & - & - & 47 & 54 & 1 & $3, a$ \\
4 & - & - & 45 & 58 & 1 & $3, a$ \\
5 & 67 & 73 & - & - & 2 & $3, b$ \\
6 & 65 & 75 & - & - & 2 & $3, b$
\end{tabular}

позволяют с гораздо меньшей точностью определить границы спектров $F_{3}$ и $F_{d}$. Определение угла $\theta_{d}$ в точке максимального изменения спектра дихроичного отношения без учета соотношения компонентов $F_{3}$ и $F_{d}$ и угла $\theta_{3}$ (который всегда больше, чем $\theta_{d}$ ) дало $\theta_{d}=49^{\circ}$ (табл. 1). Расчет спектров форм при этом значении $\theta_{d}$ и широком варьировании $\theta_{3}$ приводит к отрицательным значениям интенсивностей спектра $F_{3}$ и к выходу спектра $F_{d}$ за пределы спектра слоя. Этот результат определяет верхнюю границу возможных вариаций $\theta_{d}$ и говорит о значительном перекрывании спектров двух форм на всем участке. Резкое изменение характера спектра дихроичного отношения в области перекрытия $F_{3}$ и $F_{d}$ свидетельствует о преимущественном влиянии $\theta_{d}$ на характер спектра поглощения на этом участке. Расчет показывает, что значительное увеличение угла $\theta_{3}$ практически не влияет на вид спектров $F_{3}$ и $F_{d}$, в то время как изменение $\theta_{d}$ в пределах нескольких градусов, а также уменьшение $\theta_{3}$ и соответственно приближение его к $\theta_{d}$ заметно сказываются на характере спектров этих форм. Оценка угла наклона дипольного момента поглощения формы $F_{3}$ в точке на спектре дихроичного отношения, где $R=1$, дала значение $\theta_{3}=55^{\circ}$, которое соответствует величине некоторого усредненного угла, вклад в который в основном вносят дипольные моменты поглощения форм $F_{3}$ и $F_{d}$ с большой разностью углов наклона. Учитывая большее влияние димера на характер спектра дихроичного отношения в этой области, можно 

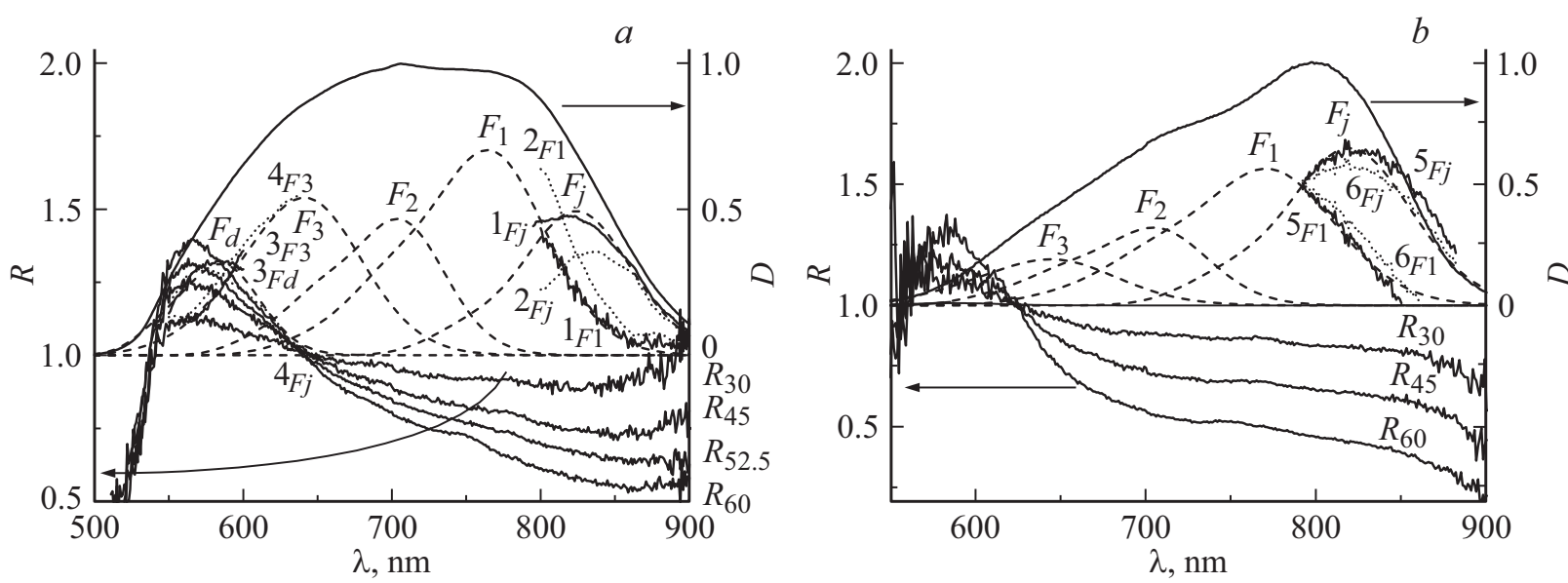

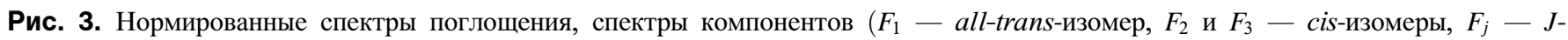
агрегат, $F_{d}$ - димер), спектры дихроичного отношения: $(a)$ необлученный образец 1 , $(b)$ облученный образец 2 . Арабскими цифрами (табл. 2) с индексами молекулярных компонентов указаны расчетные участки спектра для разных форм.
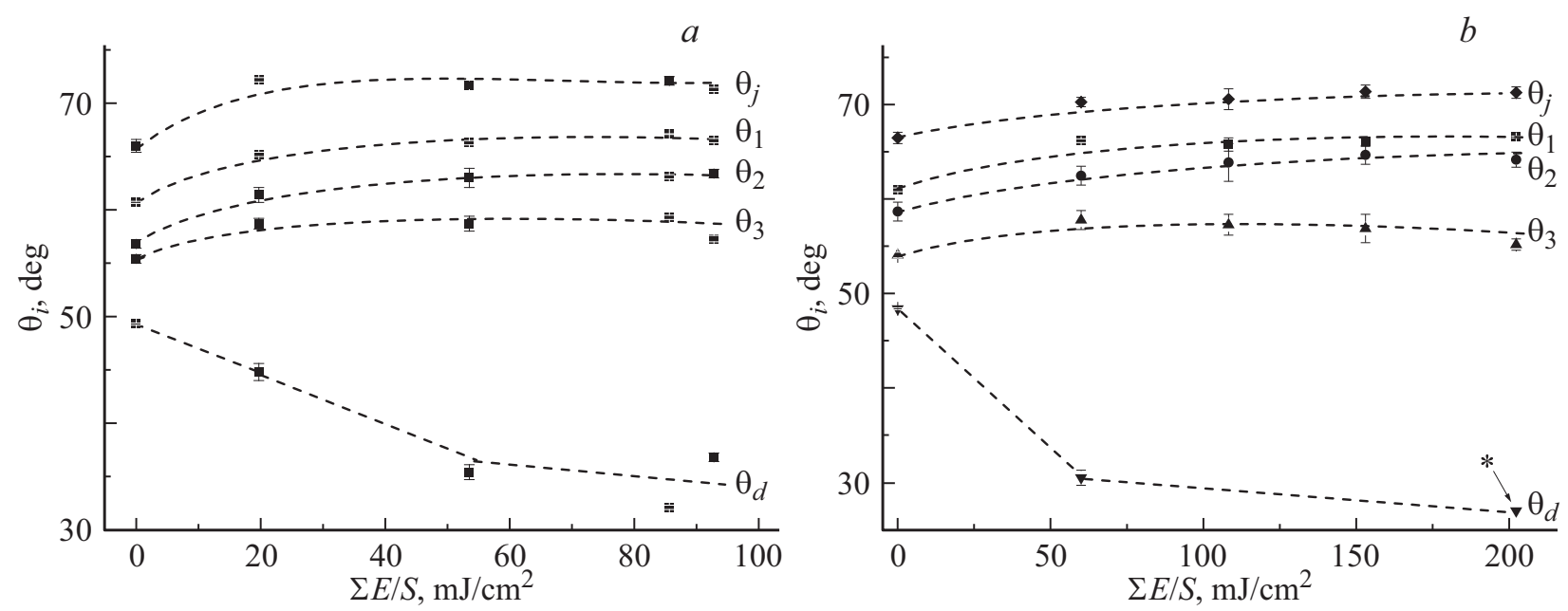

Рис. 4. Зависимости ориентации дипольных переходов молекулярных компонентов от суммарной плотности энергии лазерного возбуждения: $(a)$ образец $1,(b)$ образец 2. Звездочкой обозначена расчетная точка.

предположить, что $\theta_{3} \geq 54^{\circ}$. Характер изменений спектральной зависимости величины $R$ как необлученного, так и облученного слоев (рис. $3, a, b)$ свидетельствует о плавном уменьшении угла наклона дипольного момента перехода с поглощением мономеров от длинноволновой к коротковолновой областям спектра. Дополнительную возможность уточнить границы изменений углов $\theta_{n}$ дают результаты их оценок в процессе облучения обоих образцов импульсным лазерным излучением. На рис. $4, a, b$ показаны зависимости углов $\theta_{i}$ от плотности энергии лазерного излучения для слоев, чьи спектры поглощения представлены на рис. $3, a, b$ соответственно.

Аналогичные измерения были проведены для ряда слоев дикарбоцианиновых молекул [18]. Как видно, увеличение суммарной плотности энергии облучения $\sum E / S$ приводит в обоих слоях сначала к плавному увеличению $\theta_{n}$ мономерных форм, а затем к насыщению зависимостей $\theta_{i}=f\left(\sum E / S\right)$, где $\sum E$ - суммарная энергия облучения, а $S$ - площадь облученного образца. Сравнение результатов исследований различных образцов показало, что уровни насыщения величин $\theta_{i}$ не зависят от плотности энергии в отдельных импульсах, а только от суммарной плотности энергии облучения и начальных углов наклона ориентации молекул в необлученных образцах [24]. Как видно из сравнения рис. 4, $a$ и 4, $b$ при одних и тех же значениях начальных углов $\theta_{3}$ и суммарной плотности энергии облучения, их значения в области насыщения одинаковы в пределах ошибки измерения и больше начального $\theta_{3}$. Таким образом, можно предположить, что максимальное значение $\theta_{3}$ не превышает $58^{\circ}$. В то же время зависимости $\theta_{d}=f\left(\sum E / S\right)$ во всех исследованных образцах не достигают насыщения, а углы $\theta_{d}$ резко уменьшаются с увеличением суммарной плотности энергии облучения. Как было показано выше, начальные значения $\theta_{d}$ на рис. 4 несколько завышены. Для образца 1 при $\sum E / S \sim 20 \mathrm{~mJ} / \mathrm{cm}^{2}$ угол $\theta_{d}=45^{\circ}$, 
и поэтому для необлученного образца $45^{\circ} \leq \theta_{d} \leq 49^{\circ}$. Приведенный анализ границ возможных изменений углов $\theta_{d}$ и $\theta_{3}$ основан на расчете спектров форм в области максимального изменения спектра дихроичного отношения, где скорее всего присутствуют только две перекрывающиеся полосы форм. Однако принимая во внимание значительное перекрытие полос в коротковолновой области спектра и связанные с этим возможные ошибки, на рис. 3, $a$ приведены результаты расчета спектров в этой области с учетом возможного небольшого вклада спектра $F_{2}$. Значение $\theta_{2}=56^{\circ}$ было получено по одной точке и мало отличается от приведенных выше значений $\theta_{3}$. Хорошее совпадение участков спектров форм $F_{d}$ и $F_{3}$ с их предварительным разделением достигается при значениях углов $\theta_{d}$ и $\theta_{3}$, попадающих в допустимые интервалы, приведенные в табл. 1.

Аналогичный анализ с учетом результатов, приведенных на рис. $4, b$ для облученного образца 2 , показал, что дипольные моменты переходов с поглощением форм $F_{j}$ и $F_{1}$, чьи спектры расположены в длинноволновой области, составляют с перпендикуляром к подложке углы $\theta_{j}>71^{\circ}$ и $\theta_{1} \geq 64^{\circ}$. Наилучшее совпадение рассчитанных спектров с исходными достигается при $\theta_{j}=73^{\circ}$ и $\theta_{1}=67^{\circ}$. При этом уменьшение $\theta_{j}$ даже на $1^{\circ}$ и вариации $\theta_{1}$ в возможных границах приводит к существенному выходу спектра $F_{j}$ за пределы спектра слоя. Как и в случае необлученного образца 1, взаимным изменением углов наклона (увеличением $\theta_{j}$ и соответствующим уменьшением $\theta_{1}$ ) можно добиться совпадения рассчитанного спектра с исходным. На рис. $3, b$ показаны возможные отклонения хода спектров от исходного при небольших вариациях обоих углов в допустимых пределах.

Резкое отличие углов наклона димера от соответствующих параметров мономеров и $J$-агрегатов, а также характер зависимостей, представленных на рис. 4, может говорить о том, что дипольный момент перехода с поглощением в этих молекулах не совпадает с дипольными моментами мономеров. При облучении угол $\theta_{d}$ между направлением дипольного момента перехода с поглощением молекулы димеров и перпендикуляром к подложке уменьшается. В результате угол между перпендикуляром к подложке и плоскостью молекулы соответственно увеличивается. То есть в результате лазерного облучения положение молекул димеров, мономеров и $J$-агрегатов на подложке становится более параллельным подложке. Уменьшению угла $\theta_{d}$ в результате облучения соответствует малое поглощение димера в коротковолновой области спектра при нормальном падении $(\alpha=0)$ зондирующего излучения на образец и резкое изменение в этой области характера спектра дихроичного отношения при $\alpha=60^{\circ}$ (рис. 3). Выделение спектра димера и оценка $\theta_{d}$ в одной точке спектра в этом случае становятся достаточно неопределенными, но мы попытались оценить значение этого угла, решая задачу, обратную предыдущей. В предположении правильности предварительного выделения коротковолнового участка спектра мономера $F_{3}$ и при варьировании величин $\theta_{d}$ и $\theta_{3}$ было получено $\theta_{d} \leq 30^{\circ}$. Этот результат не противоречит характеру зависимостей $\theta_{d}$ от плотности энергии лазерного излучения, показанному на рис. $4, b$.

\section{Выводы}

1. Для выделения спектров отдельных молекулярных компонентов из спектров поглощения молекулярных слоев оказывается полезным сочетание разных независимых методик: измерения спектров концентрационных серий слоев в совокупности с независимым измерением их спектров дихроичного отношения.

2. Сочетание этих методов дает возможность оценить углы наклона дипольных моментов перехода с поглощением мономеров и ассоциированных форм молекул как в отдельных точках, так и на протяженных участках спектра поглощения этих форм.

3. Проведенные расчеты подтверждают тенденцию все более горизонтального расположения молекулярных форм слоя в процессе лазерного облучения. Отметим,что этот результат коррелирует с результатами исследования облученных и необлученных слоев методом атомно-силовой микроскопии [19]. Было выяснено, что облучение уменышает высоту неоднородностей и сглаживает поверхность слоя, что связано с более горизонтальной ориентацией компонентов.

4. Характер изменений спектра дихроичного отношения в его коротковолновой части и соответствующее изменение углов дипольного момента перехода с поглощением подтверждают димерную природу молекулярной формы, поглощающей в этой спектральной области.

\section{Список литературы}

[1] Tang C.W., VanSlyke S.A. // J. Appl. Phys. Lett. 1987. V. 51. P. 913. doi 10/1063/1.98799

[2] Fou A.C., Onitsuka O., Ferreira M., Rubner M.F., Hsieh B.R. // Appl. Phys. 1996. V. 79. P. 7051. doi 10/1063/1.362421

[3] Tao S., Zhou Y., Lee C.S., Zhang X., Lee S.T. // Chem. Mater. 2010. V. 22. P. 2138. doi $10 / 1021 / \mathrm{cm} 100100 \mathrm{w}$

[4] Sun N., Zhao Y., Zhao F., Chen Y., Yang D., Chen J., Ma D. // Appl. Phys. Lett. 2014. V. 105. P. 013303. doi 10/1063/1.4890217

[5] O'Regan B., Gratzel M. // Nature. 1991. V. 353. P. 737. doi 10/1038/353737 aO

[6] Zhou Q., Hou Q., Zheng L., Deng X., Yu G., Cao Y. // Appl. Phys. Lett. 2004. V. 84. P. 1653. doi 10/1063/1.1667614

[7] Wada A., Nishida J., Maitani M.M., Wada Y., Yamashita Y. // Chem. Lett. 2014. V. 43. N 3. P. 296. doi 10/1246/cl.130931

[8] Liu X., Tavares L., Osadnik A., Lausen J.L., Kongsted J., Lutzen A., Rubahn H.G., Kjelstrup-Hansen J. // Org. Electron. 2014. V. 15. N 6. P. 1273. doi 10/1016/j.orgel.2014.02.023

[9] Mei J., Diao Y., Appleton A.L., Fang L., Bao Z. // J. Am. Chem. Soc. 2013. V. 135. N 18. P. 6724. doi 10/1021/ja400881n

[10] Shindy H.A. // Dyes Pigm. 2017. V. 145. P. 505. 
[11] Guarin C.A., Villabona-Monsalve J.P., Lopez-Arteaga R., Peon J. // J. Phys. Chem. B. 2013. V. 117. N 24. P. 7352. doi $10 / 1021 / \mathrm{p} 400278 \mathrm{t}$

[12] Kojima O., Hamano S., Kita T., Wada O. // J. Appl. Phys. 2011. V. 110. N 8. P. 083521. doi 10.1063/1.3653228

[13] Kaliteevskaya E.N., Krutyakova V.P., Razumova T.K., Roshal A.D., Starovoytov A.A. // Opt. Quant. Electron. 2017. V. 49. P. 32 (1-8); Kaliteevskaya E., Krutyakova V., Razumova T., Roshal A., Starovoytov A. [Электронный ресурс] Режим доступа: https://link.springer.com/journal/11082

[14] Калитеевская Е.Н., Крутякова В.П., Разумова Т.К., Старовойтов А.А. // Опт. и спектр. 2016. Т. 120. № 3. C. 508; Kaliteevskaya E.N., Krutyakova V.P., Razumova T.K., Starovoitov A.A.// Opt. Spectrosc. 2016. V. 120. N 3. P. 482. doi 10.7868/S0030403416030120

[15] Калитеевская Е.Н., Крутякова В.П., Разумова Т.К., Старовойтов А.А. // Опт. и спектр. 2011. Т. 110. № 3. C. 398; Kaliteevskaya E.N., Krutyakova V.P., Razumova T.K., Starovoytov A.A. // Opt. Spectrosc. 2011. V. 110. N 3. P. 363. doi 10.1134/S0030400X11030118

[16] Калитеевская Е.Н., Крутлкова В.П., Разумова Т.К., Старовойтов А.А. // Опт. и спектр. 2012. Т. 112. № 2. C. 231; Kaliteevskaya E.N., Krutyakova V.P., Razumova T.K., Starovoytov A.A. // Opt. Spectrosc. 2012. V. 112. N`2. P. 206. doi 10.1134/S0030400X12020142

[17] Mishra A., Behera R.K., Behera P.K., Mishra B.K., Behera G.B. // Chem. Rev. 2000. V. 100. N 6. P. 1973. doi 10/1021/cr990402t

[18] Старовойтов А.А., Разумова Т.К., Калитеевская Е.Н., Крутякова В.П. // Оптический журнал. 2014. Т. 81. № 5. C. 80; Starovoytov A.A., Razumova T.K., Kaliteevskaya E.N., Krutyakova V.P. // J. Opt. Technol. 2014. V. 81. N 5. P. 289. doi 10.1364/JOT.81.000289

[19] Воронин Ю.М., Калитеевская Е.Н., Крутякова В.П., Разумова Т.К., Старовойтов А.А., Щедрин П.В. // Оптический журнал. 2011. Т. 78. № 8. С. 72; Kaliteevskaya E.N., Krutyakova V.P., Razumova T.K., Starovoitov A.A., Shchedrin P.V., Voronin Yu.M. // J. Opt. Technol. 2011. V. 78. N 8. P. 524. doi 10.1364/JOT.78.000524

[20] Kasha M., Rawls H.R., Ashraf El-Bayoumi M. // Pure Appl. Chem. 1965. V. 11. N 3-4. P. 371. doi 10.1351/pac19651103037

[21] Emerson E.S., Conlin M.A., Rosenoff A.E., Norland K.S., Rodriguez H., Chin D., Bird G.R. // J. Phys. Chem. 1967. V. 71. N 8. P. 2396. doi $10.1021 / \mathrm{j} 100867 \mathrm{a} 003$

[22] Chambers R.W., Kajiwara T., Kearns D.R. // J. Phys. Chem. 1974. V. 78. N 4. P. 380. doi 10.1021/j100597a012

[23] Starovoytov A.A., Kaliteevskaya E.N., Krutyakova V.P., Razumova T.K., Toropov N.A. // Proc. SPIE. 2012. V. 8435. P. $84352 \mathrm{G}(1-6)$. doi $10.1117 / 12.926866$

[24] Starovoytov A., Kaliteevskaya E.N., Krutyakova V.P., Razumova T.K. // Proc. SPIE. 2015. V. 9549. P. 95490Y (1-6). doi $10.1117 / 12.2192604$ 